\title{
Oligohydramnios sequence and a variant of Dandy-Walker syndrome
}

\author{
Prasad Yeshwant Deshmukh*, Shaifali Kundan Patil, B. G. Boricha
}

Department of Obstetrics \& Gynaecology, MGM Medical College, Navi Mumbai, Maharashtra, India

Received: 21 August 2014

Accepted: 17 September 2014

\section{*Correspondence:}

Dr. Prasad Yeshwant Deshmukh,

E-mail: drpydeshmukh@gmail.com

Copyright: $\odot$ the author(s), publisher and licensee Medip Academy. This is an open-access article distributed under the terms of the Creative Commons Attribution Non-Commercial License, which permits unrestricted non-commercial use, distribution, and reproduction in any medium, provided the original work is properly cited.

\begin{abstract}
Dandy-Walker syndrome is frequently associated with disorders of other areas of the central nervous system, including absence of the area made up of nerve fibers connecting the two cerebral hemispheres (corpus callosum) and malformations of the heart, face, limbs, fingers and toes. In the diagnostic workup, ultrasound is reliable and accurate for making the diagnosis. For diagnostic purposes and confirmation of diagnosis, foetal MRI in utero also has been advocated. Further confirmation may be done with autopsy of neonate after birth. Most of the pregnancies are terminated after antenatal diagnosis on anomaly scan. However if born alive the survival depends on associated anomalies in other systems
\end{abstract}

Keywords: Dandy-Walker syndrome, Oligohydramnios sequence, Anomaly scan, Fetal MRI, Fetal autopsy, Poor prognosis

\section{INTRODUCTION}

Oligohydramnios sequence consists of lung hypoplasia, facial deformity, renal agenesis, which has a lethal outcome. Hydrocephalus, herniated cerebellar vermis (variant of Dandy Walker syndrome) has incidence of 1 in 30000. These deformities are recorded as incompetent with life, which are associated with many chromosomal aneuploidies and are associated with many syndromes.

Dandy-Walker malformation is a rare abnormality of the Central Nervous System (CNS) with a reported incidence of 1 in 25000-35000 live births and a slight female predominance. It accounts for $1-4 \%$ of cases of antenatally detected hydrocephalus. Dandy Walker variant forms part of the spectrum of Dandy Walker malformation. It is characterized by partial agenesis of the vermis, resulting in communication between the fourth ventricle and the cisterna magna.

\section{CASE REPORT}

A case of 26 year old, gravida 3, para 2, living issue 1, death 1 who came with complaints of pain in abdomen (uterine activity 2-3/10 minutes lasting for 20-30 seconds) and leaking of clear fluid since 2 hours. There was no history of trauma or decreased foetal movements. There was no history of antenatal registration to any of midwifery unit and no previous ultrasound report was available for pregnancy dating. She had H/o consanguineous marriage and 1 previous normal delivery 3 years ago and 1 preterm delivery of malformed baby 1 year ago. Details of these malformations were not known.

On examination, she was found to be vitally stable. Per abdominally, she was 28-30 weeks of gestation with regular FHR of $148 / \mathrm{min}$. Per vaginally, she was $3 \mathrm{~cm}$ dilated; $50 \%$ effaced; with absent membranes with head at ischial spine. Patient was thoroughly investigated. Haemoglobin of $9.3 \mathrm{gm} \%$ and B +ve blood group. Ultrasonogram was done and baby was found to have multiple congenital anomalies in the form of polycystic 
kidneys and bladder, absent diaphragm, hydrocephalus, hypoplastic lungs, cystic hygroma, ventricular septal defect and echogenic bowels with severe oligohydramnios.

She delivered a female baby of 1080 gm. Neonatal APGAR score was 3 and 5 on one and five minute postdelivery. Baby was taken to NICU, however did not survive. Cause of death was evident on antenatal ultrasonogram, which showed multiple anomalies, not compatible with life.

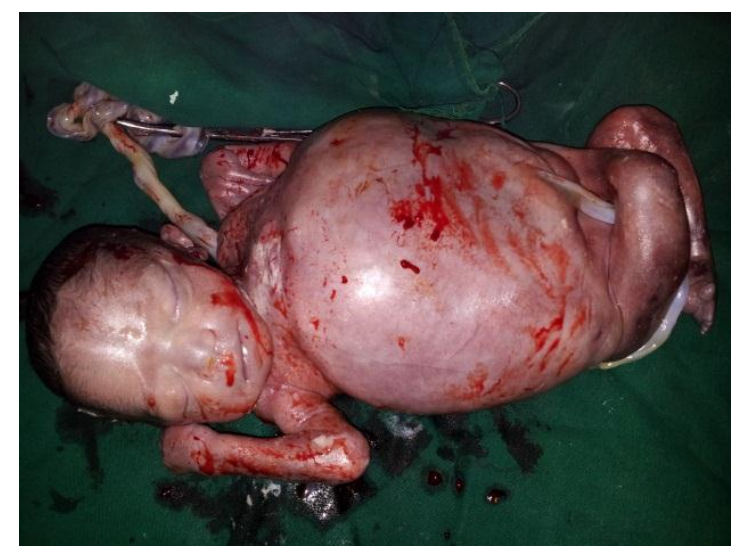

Figure 1: Baby of Dandy-Walker malformation showing gross ascites and clinical picture of foetal hydrops.

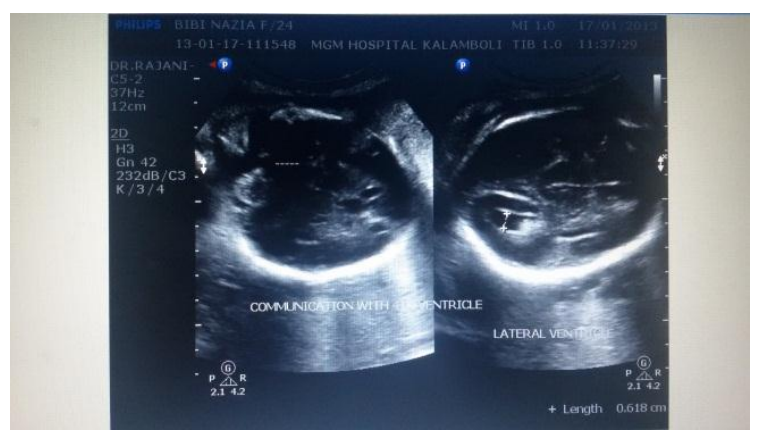

Figure 2: Ultrasound showing foetal hydrocephalus and a narrowed connection to fourth ventricle.

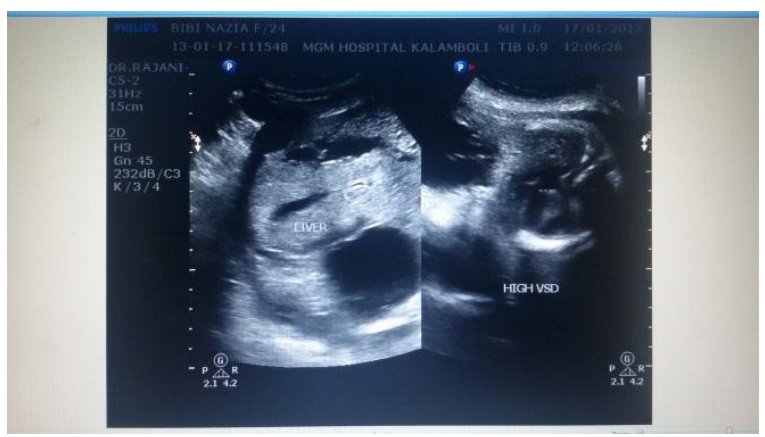

Figure 3: Ultrasound plate of anomaly scan showing foetal cardiomegaly and high ventricular septal defect.

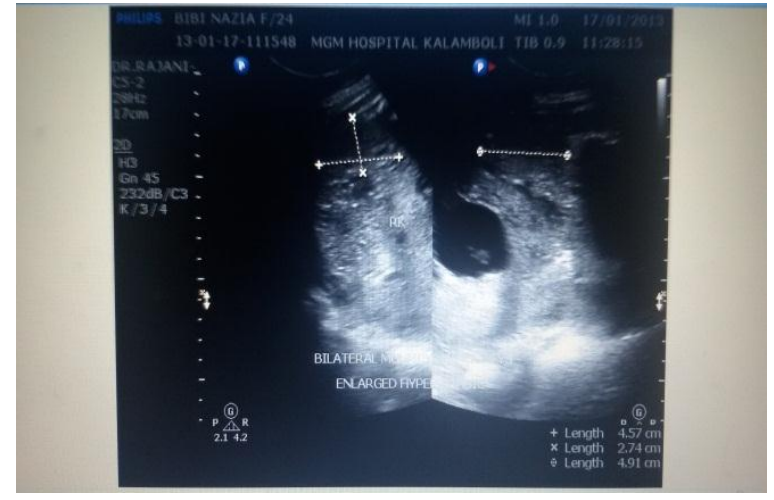

Figure 4: Ultrasound picture of bilateral multicystic dysplastic kidneys and aorta that is pushed posteriorly.

\section{DISCUSSION}

There are several abnormalities associated with DandyWalker malformation. The most common are ventriculomegaly (70-90\%) and cardiac defects. Midline defects such as agenesis of the corpus callosum, holoprosencephaly, encephalocele, polycystic kidney disease and facial dysmorphism etc. have been variously reported to be associated with this condition. The incidence rate of karyotypic abnormalities, mainly trisomy 18 and 13 and triploidy, is up to $40 \%$. The Dandy-Walker variation is also part of more than 50 genetic syndromes. The outcome following prenatal diagnosis varies widely. A large proportion of pregnancies will be terminated owing to the identification of associated abnormalities and the karyotypic defects. Overall mortality rates of $12-50 \%$ have been reported; associated congenital abnormalities contribute to $83 \%$ of postnatal deaths and subnormal intelligence is reported in $40-70 \%$ of cases. The isolated Dandy Walker variant abnormality has the highest incidence of survival, and there are reported cases of people who have had Dandy Walker variant their entire lives without any symptoms. ${ }^{2}$

The syndrome can appear dramatically or develop unnoticed. Symptoms, which often occur in early infancy, include slow motor development and progressive enlargement of the skull. In older children, symptoms of increased intracranial pressure such as irritability and vomiting, and signs of cerebellar dysfunction such as unsteadiness, lack of muscle coordination, or jerky movements of the eyes may occur. Other symptoms include increased head circumference, bulging at the back of the skull, problems with the nerves that control the eyes, face and neck, and abnormal breathing patterns. ${ }^{3}$

Treatment for individuals with Dandy-Walker syndrome generally consists of treating the associated problems, if needed. A surgical procedure called a ventriculoperitoneal shunt may be required to drain off excess fluid within the brain. This will reduce intracranial pressure and help control swelling. Parents of children with 
Dandy-Walker syndrome may benefit from genetic counseling if they intend to have more children. ${ }^{4}$

The effect of Dandy-Walker syndrome not associated with any other systemic anomalies is viable, their intellectual development is variable, some children have normal cognition and others never achieve normal intellectual development even when the excess fluid buildup is treated early and correctly. Longevity depends on the severity of the syndrome and associated malformations. However the presence of multiple congenital defects may shorten life span or cause foetal death in utero. ${ }^{4}$

The concept of small family norm, which is percolating in young and educated generation makes it important to have a non-challenging baby. To avoid sudden instant anguish of these parents, we present this case to highlight the fact that anomaly scan along with biochemical markers is an integral part of antenatal follow up in early trimester around 18-20 weeks, so that if any anomaly is detected, it is possible to abort the baby within the period approved by MTP act. Also it is possible to take opinion from paediatrician and paediatric surgeon. This team of doctors can put positive inputs in management of such babies.
Funding: No funding sources Conflict of interest: None declared

Ethical approval: Not required

\section{REFERENCES}

1. Griffiths PD, Paley MN, Whitby EH. Post-mortem MRI as an adjunct to fetal or neonatal autopsy. Lancet. 2005;365:1271-3.

2. Osenbach RK, Menezes AH. Diagnosis and management of the Dandy-Walker malformation: 30 years of experience. Paediatr Neurosurg. 1992;18:179-89.

3. Bromley B, Nadel AS, Pauker S, Estroff JA, Benacerraf BR. Closure of the cerebellar vermis: evaluation with second trimester US. Radiology. 1994;193:761-3.

4. Lavanya T, Cohen M, Gandhi SV. A case of a Dandy-Walker variant: the importance of a multidisciplinary team approach using complementary techniques to obtain accurate diagnostic information. Br J Radiol. 2008;81:245-7.

DOI: $10.5455 / 2320-1770 . i j r \operatorname{cog} 20141246$

Cite this article as: Deshmukh PY, Patil SK, Boricha

BG. Oligohydramnios sequence and a variant of Dandy-Walker syndrome. Int J Reprod Contracept Obstet Gynecol 2014;3:1107-9. 